\title{
Investigation of Substrate Swell-Induced Defect Formation in Suspended Graphene upon Helium Ion Implantation
}

Shixuan He, ${ }^{\dagger}, \star, \|$ Wanyi Xie,,${ }^{\dagger, \S, \|}$ Yongna Zhang, ${ }^{\dagger}$ Shaoxi Fang, ${ }^{\dagger, \S}$ Daming Zhou, ${ }^{\dagger}$

Zhiyou Zhang, ${ }^{\ddagger}$ Jinglei Du ${ }^{\ddagger}$ Chunlei Du ${ }^{*}, \star$ and Deqiang Wang ${ }^{*}, \dagger, \S$

${ }^{\dagger}$ Chongqing Institute of Green and Intelligent Technology, Chinese Academy of

Sciences, Chongqing, 400714, P. R. China

$\$$ College of Physics, Sichuan University, Chengdu, Sichuan, 610065, P. R. China

$\S$ Chongqing School, University of Chinese Academy of Sciences, Chongqing,

400714, P. R. China

*Email: cldu@yznu.edu.cn

*Email: dqwang@cigit.ac.cn

" These authors contributed equally to this work. 
(a)

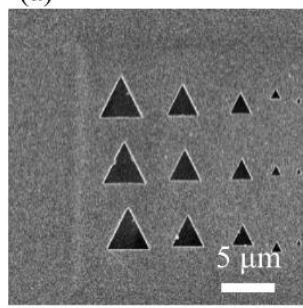

(b)

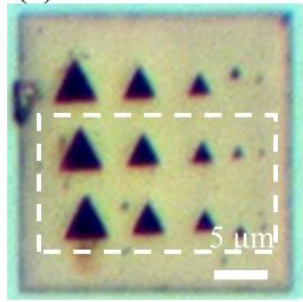

(c)

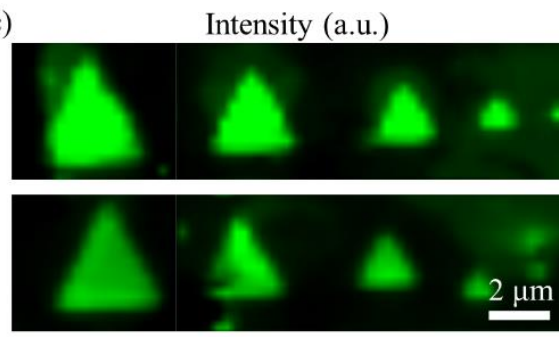

(d)
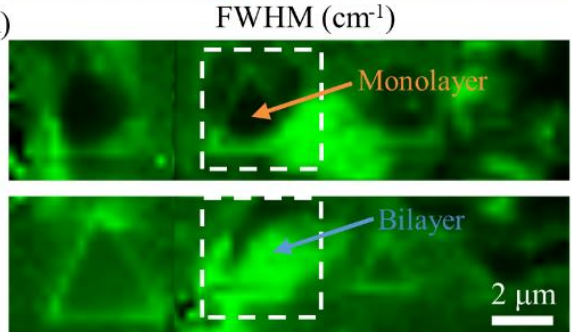
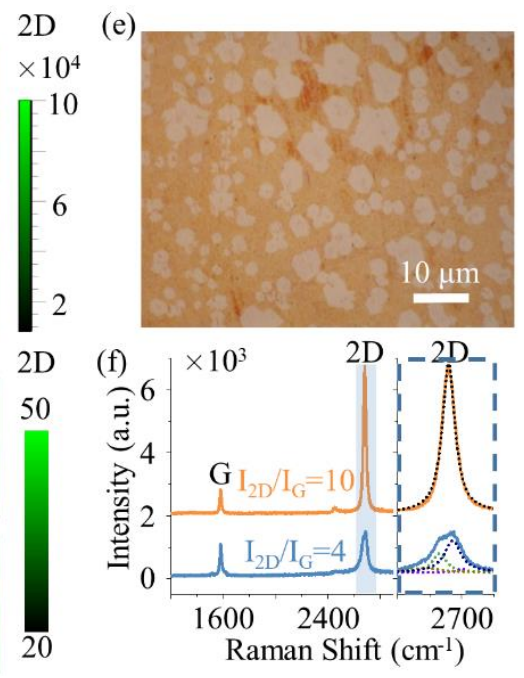

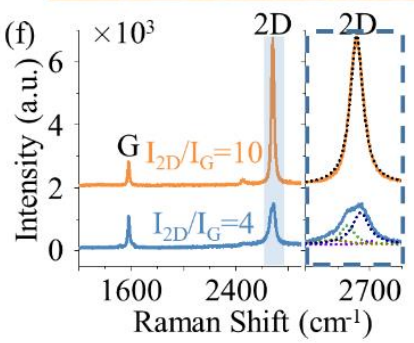

Figure S1. Suspended graphene membrane and its Raman characteristics. (a) FIB micrograph of $\mathrm{Si}_{3} \mathrm{~N}_{4} / \mathrm{SiO}_{2}$ substrate with triangular hole structures. (b) Optical micrograph of bilayer graphene on the $\mathrm{Si}_{3} \mathrm{~N}_{4} / \mathrm{SiO}_{2}$ substrate with triangular hole structures. Raman mapping of intensities (c) and FWHMs (d) at 2D peak of bilayer graphene on the $\mathrm{Si}_{3} \mathrm{~N}_{4} / \mathrm{SiO}_{2}$ substrate with triangular hole structures, each of the Raman mapping was carried out in the areas specified by the white dashed boxes in (b). (e) Optical micrograph of as-grown bilayer graphene on copper foil. (f) Representative Raman spectra of suspended monolayer and bilayer graphene (mark as arrows in d) and their fitting results of 2D peak with Lorentzian functions.

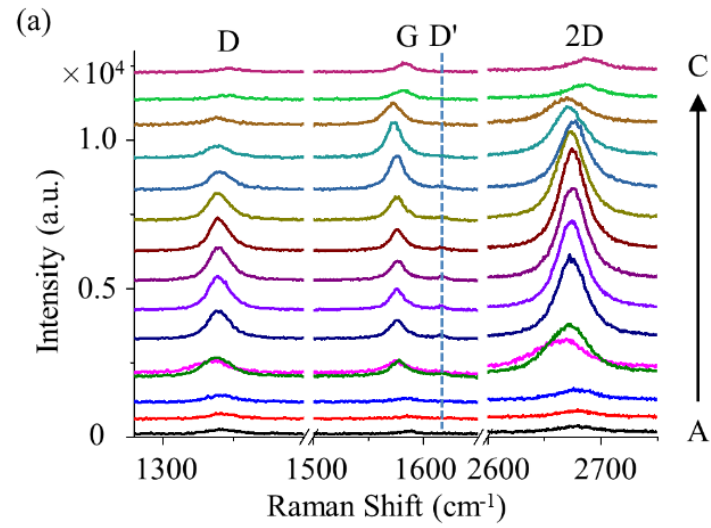

(b)

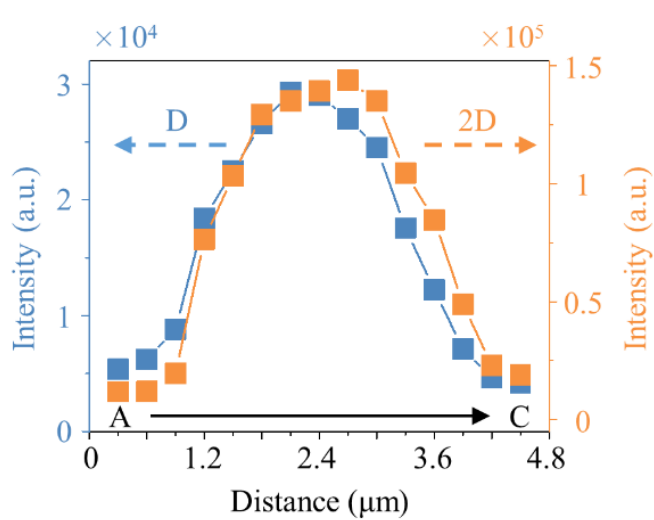

Figure S2. Raman spectra and their intensities at D and 2D peaks of graphene sample 
on the $\mathrm{Si}_{3} \mathrm{~N}_{4} / \mathrm{SiO}_{2}$ substrate with a triangular hole structure after helium ion implantation. (a) Raman spectra of graphene sample from A to C in Figure 1. (b) Raman intensities at D and 2D peaks of graphene sample from A to C in Figure 1.

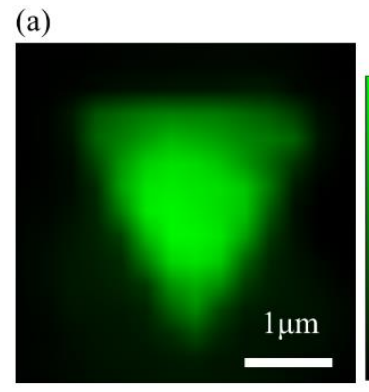

(d)

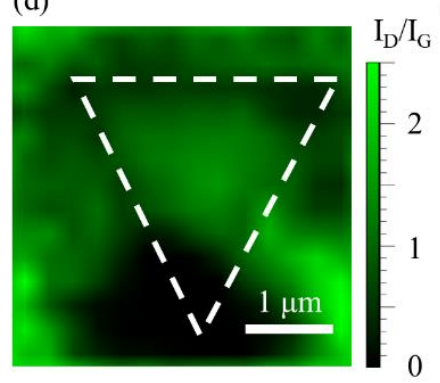

(b)

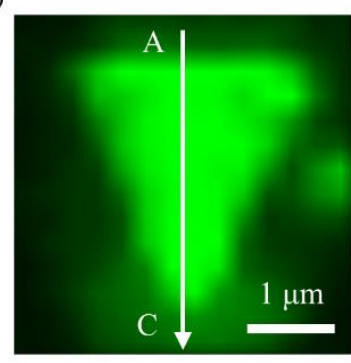

(e)

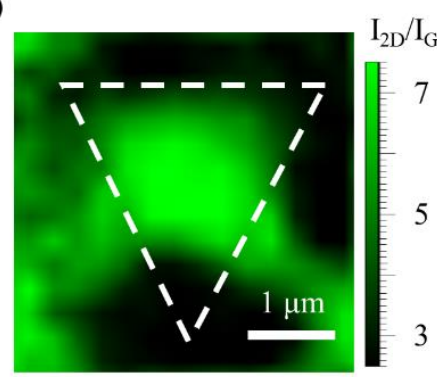

(c)
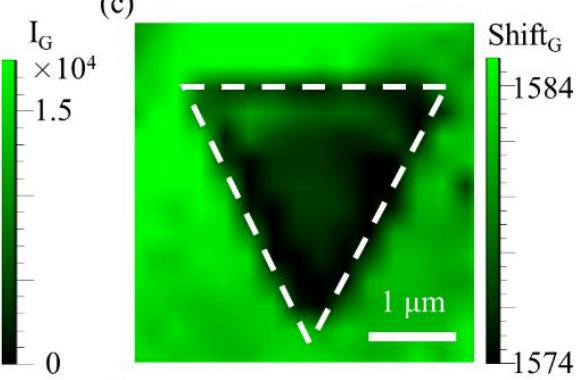

(f)

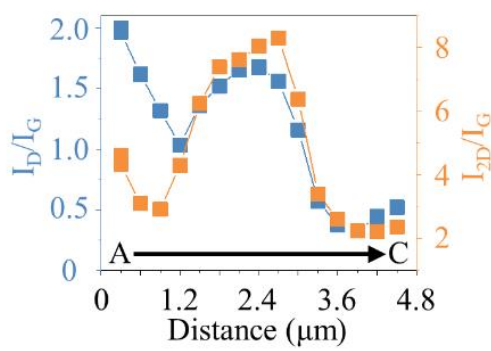

Figure S3. Raman characteristics of graphene membrane on the $\mathrm{Si}_{3} \mathrm{~N}_{4} / \mathrm{SiO}_{2}$ substrate with a triangular hole structure after helium ion implantation. Raman intensities at (a) 2D and (b) G peaks and (c) Raman shifts at G peak of graphene sample with mapping technology. Relative Raman intensities at (d) D and (e) 2D peaks of graphene on the $\mathrm{Si}_{3} \mathrm{~N}_{4} / \mathrm{SiO}_{2}$ substrate with a triangular hole structure. (f) Relative Raman intensities at the $\mathrm{D}$ and $2 \mathrm{D}$ peaks of graphene from $\mathrm{A}$ to $\mathrm{C}$ on the $\mathrm{Si}_{3} \mathrm{~N}_{4} / \mathrm{SiO}_{2}$ substrate with a triangular hole structure. 
(a)

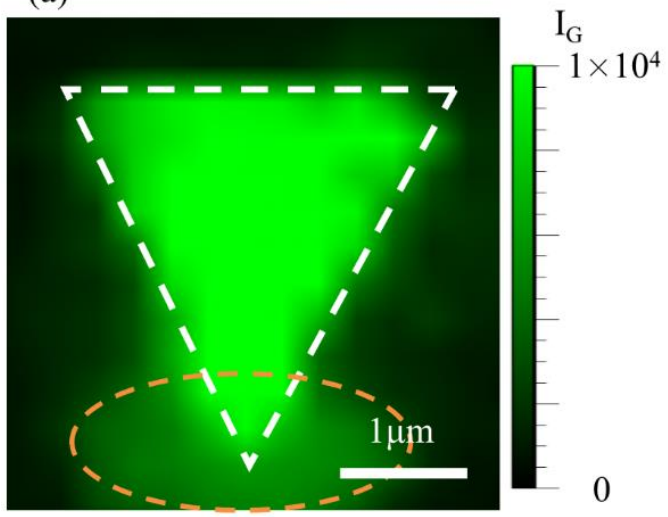

(b)

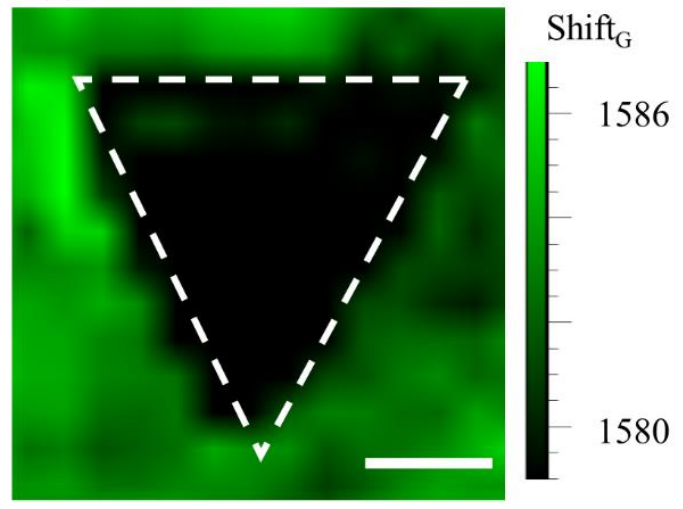

Figure S4. Raman intensities and shifts at $G$ peak of monolayer graphene on the $\mathrm{Si}_{3} \mathrm{~N}_{4} / \mathrm{SiO}_{2}$ substrate with a triangular hole structure before helium ion implantation.

(a)

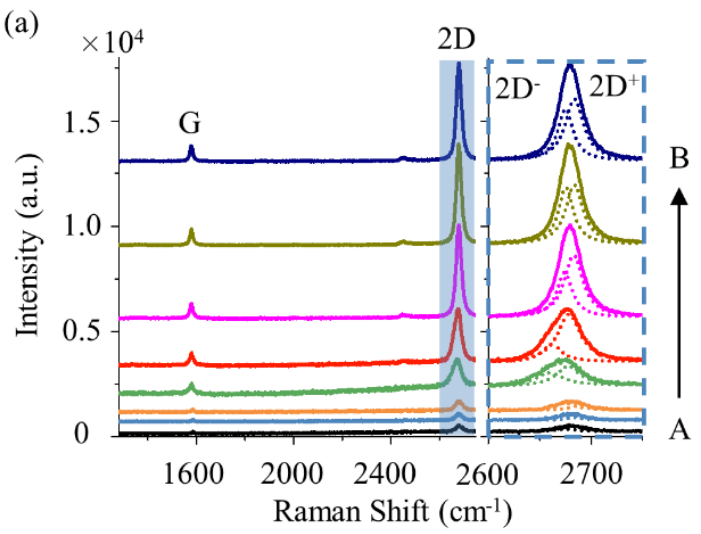

(b)

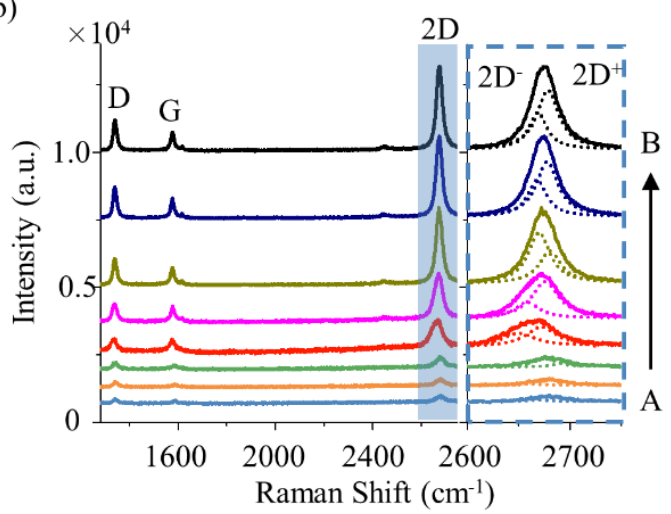

Figure S5. Raman characteristics of graphene at the edge of a triangular hole structure before and after helium ion implantation. Raman spectra of graphene at the white dotted line in Figure $2 b(a)$ before and (b) after helium ion implantation. 
(a)

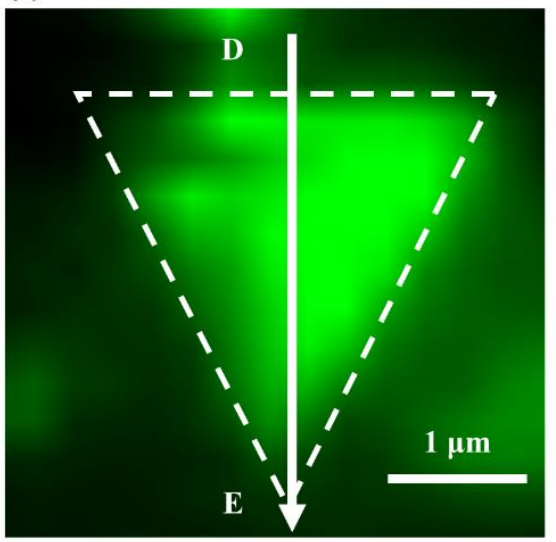

(c)

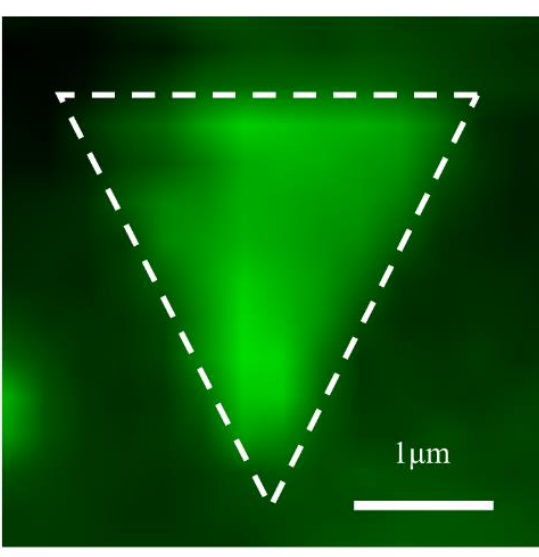

(e)

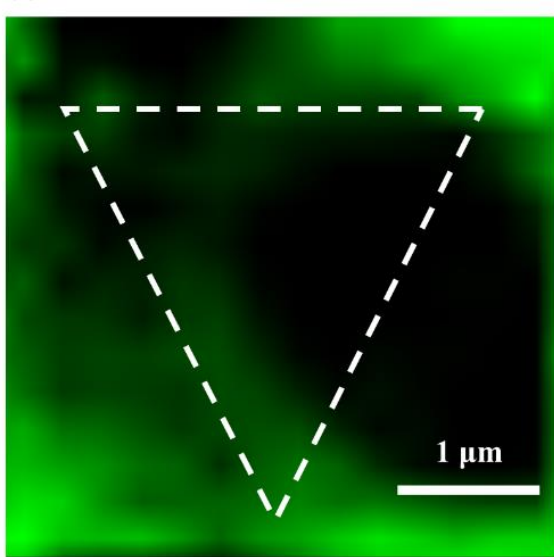

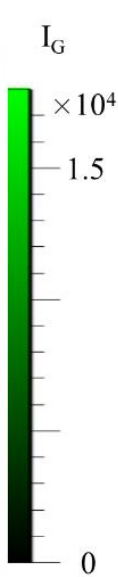

$\mathrm{I}_{\mathrm{D}}$

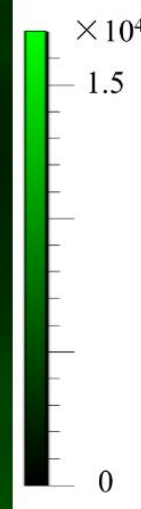

0

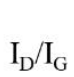

$\mathrm{I}_{\mathrm{D}} / \mathrm{I}_{\mathrm{G}}$

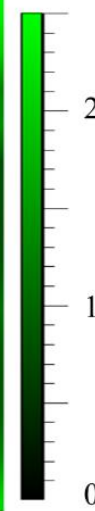

(b)

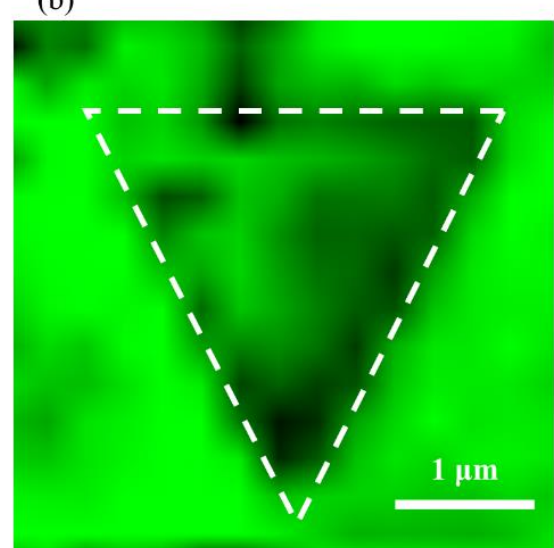

Shift $_{\mathrm{G}}$

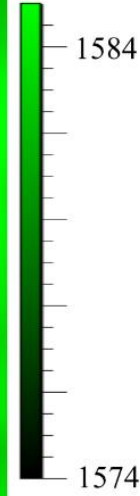

(d)

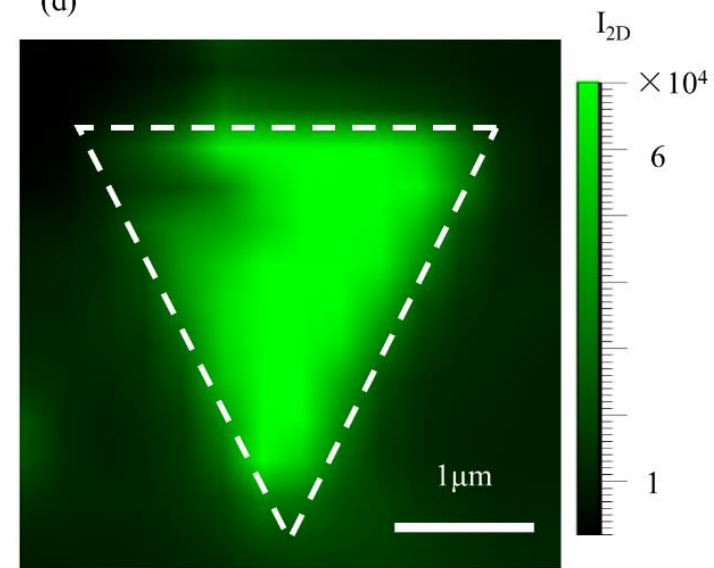

(f)

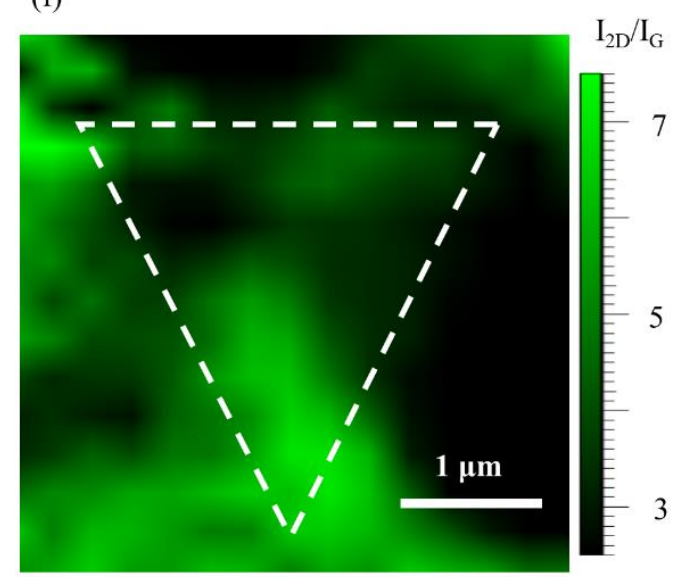

Figure S6. Raman mapping images of bilayer graphene on the $\mathrm{Si}_{3} \mathrm{~N}_{4} / \mathrm{SiO}_{2}$ substrate with a triangular hole structure after helium ion implantation. (a) Raman intensities and (b) shifts at G peak of bilayer graphene with mapping technology. Raman intensities at (c) D and (d) 2D peak of bilayer graphene with mapping technology. Relative intensities (e) $I_{\mathrm{D}} / I_{\mathrm{G}}$ and (f) $I_{2 \mathrm{D}} / I_{\mathrm{G}}$ of bilayer graphene with mapping technology. 
(a)

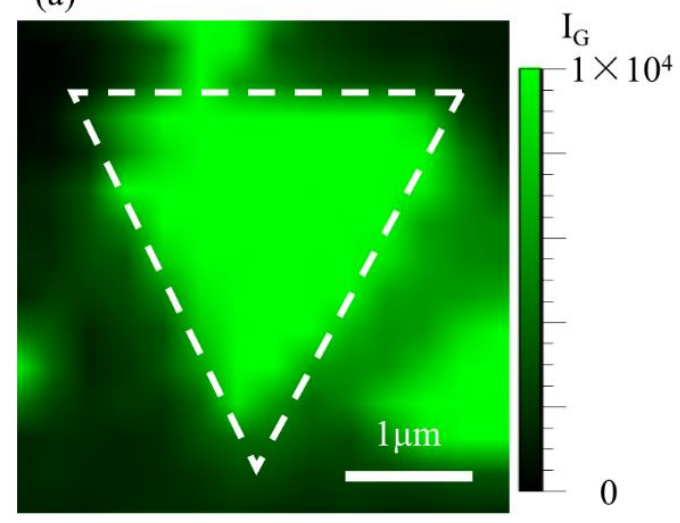

(b)

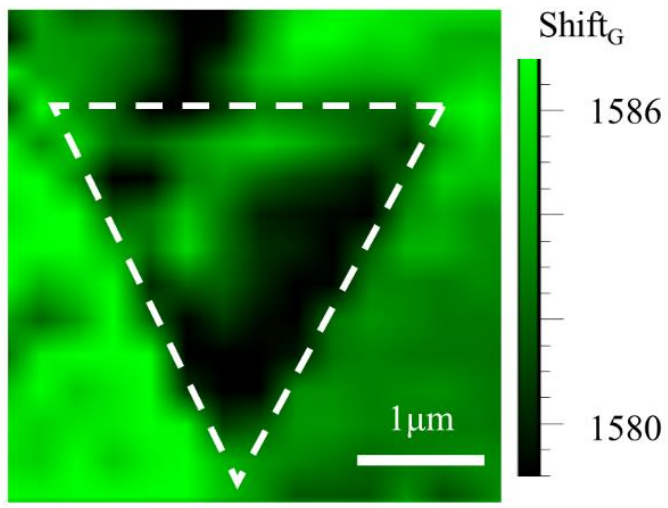

Figure S7. Raman intensities and shifts at $\mathrm{G}$ peak of bilayer graphene on the $\mathrm{Si}_{3} \mathrm{~N}_{4} / \mathrm{SiO}_{2}$ substrate with a triangular hole structure before helium ion implantation.

(a)

(c)
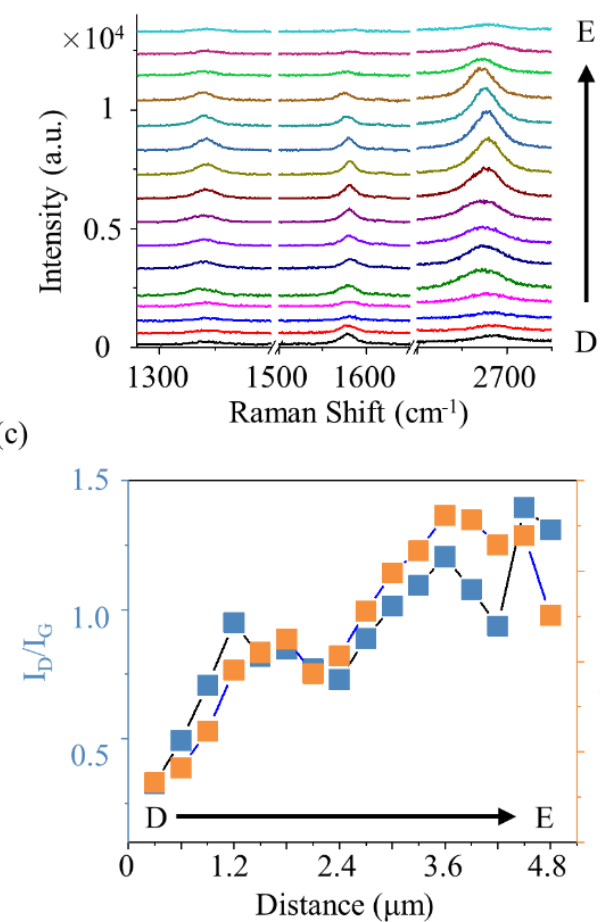

(b)

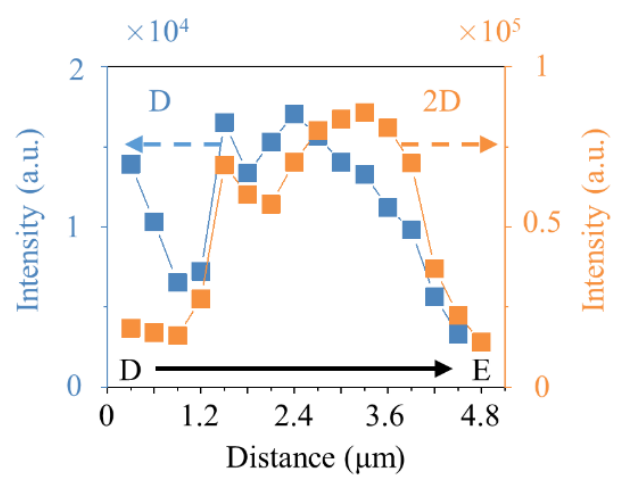

(d)

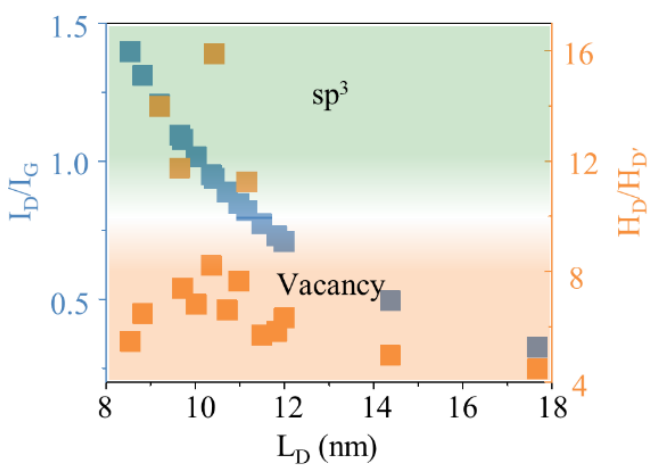

Figure S8. Raman characteristics of graphene with a bilayer domain from D to E in Figure $\mathrm{S} 5 \mathrm{a}$ on the $\mathrm{Si}_{3} \mathrm{~N}_{4} / \mathrm{SiO}_{2}$ substrate with a triangular hole structure after helium ion implantation. (a) Raman spectra of suspended bilayer graphene. (b) Raman intensities of suspended bilayer graphene at the white dotted line in Figure S6a. (c) Relative Raman intensities at D peak and 2D peak of graphene at the white dotted line in (c). (d) 
The calculated average distance between defects $L_{\mathrm{D}}$ correlates with relative intensities $I_{\mathrm{D}} / I_{\mathrm{G}}$ and relative heights $H_{\mathrm{D}} / H_{\mathrm{D}^{\prime}}$

(a)
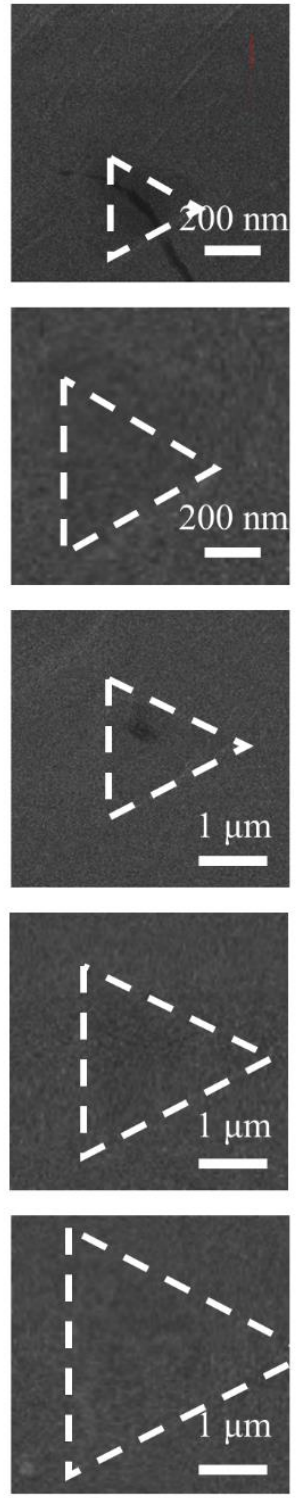

(b)
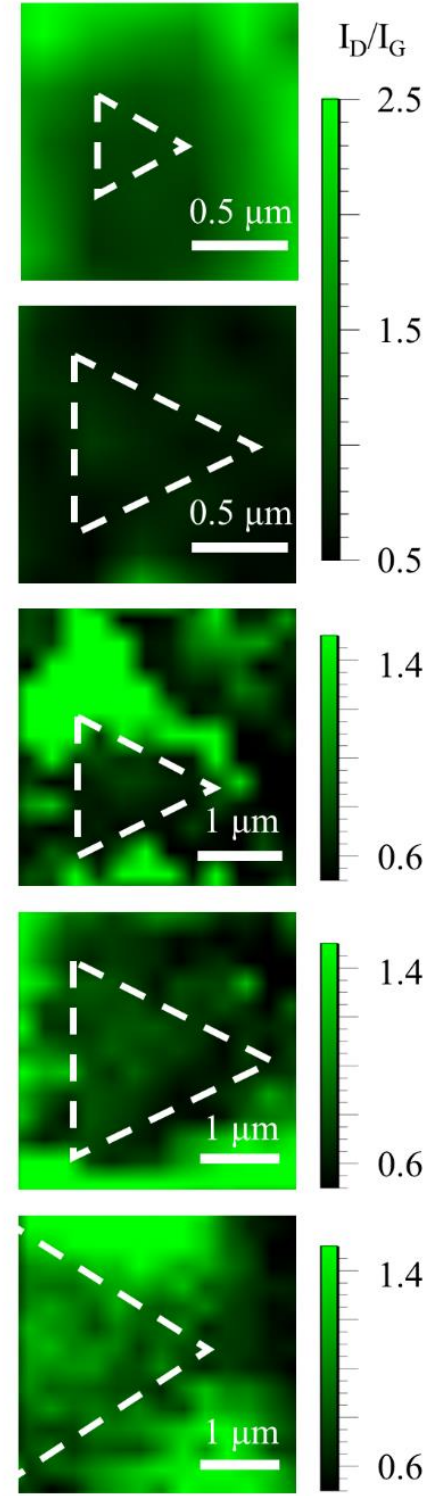

(c)
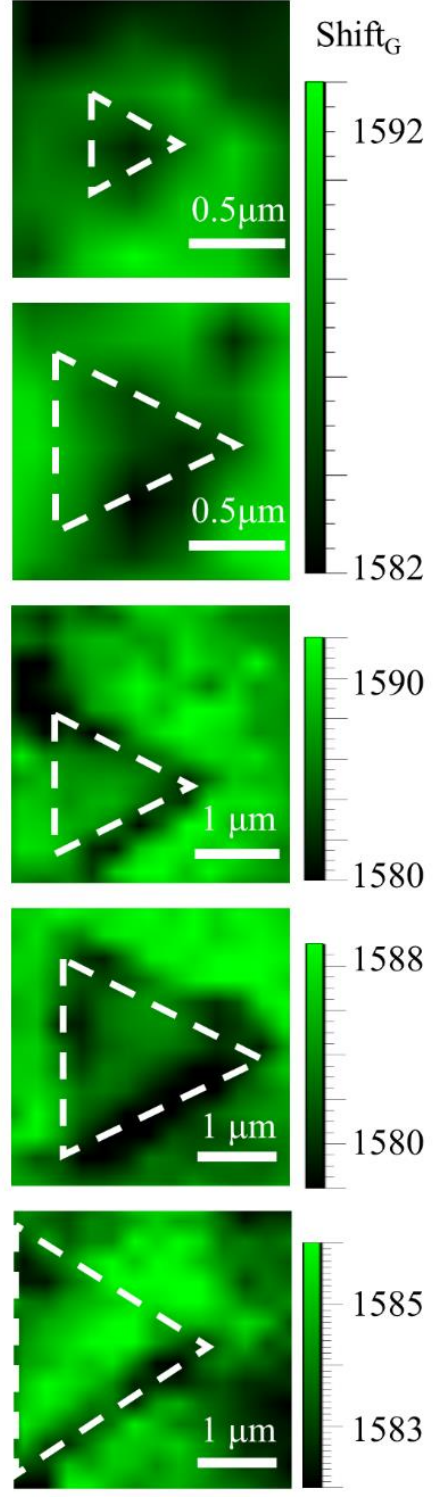

Figure S9. The suspended graphene on the $\mathrm{Si}_{3} \mathrm{~N}_{4} / \mathrm{SiO}_{2}$ substrate with different size triangular hole structures and theirs Raman characteristics. (a) HIM images of graphene membrane (dwell time: $2 \mu \mathrm{s}$ ). (b) Relative Raman intensities $I_{\mathrm{D}} / I_{\mathrm{G}}$ of graphene membrane. (c) Raman shifts at G peak of graphene membrane. 


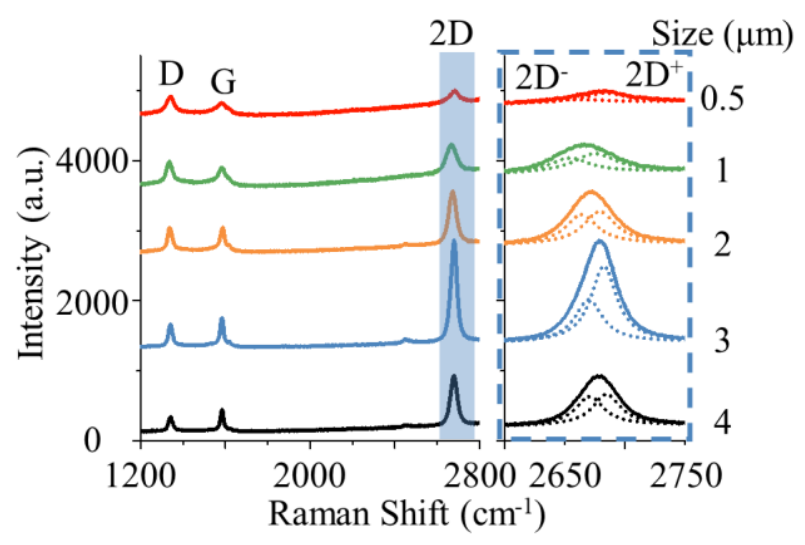

Figure S10. The average spectra of graphene membrane suspended on the $\mathrm{Si}_{3} \mathrm{~N}_{4} / \mathrm{SiO}_{2}$ substrate with different size triangular hole structures after helium ions implantation, the right part is the fitting results for 2D peak of graphene membrane, it was well fitted with two Lorentzian type peaks.
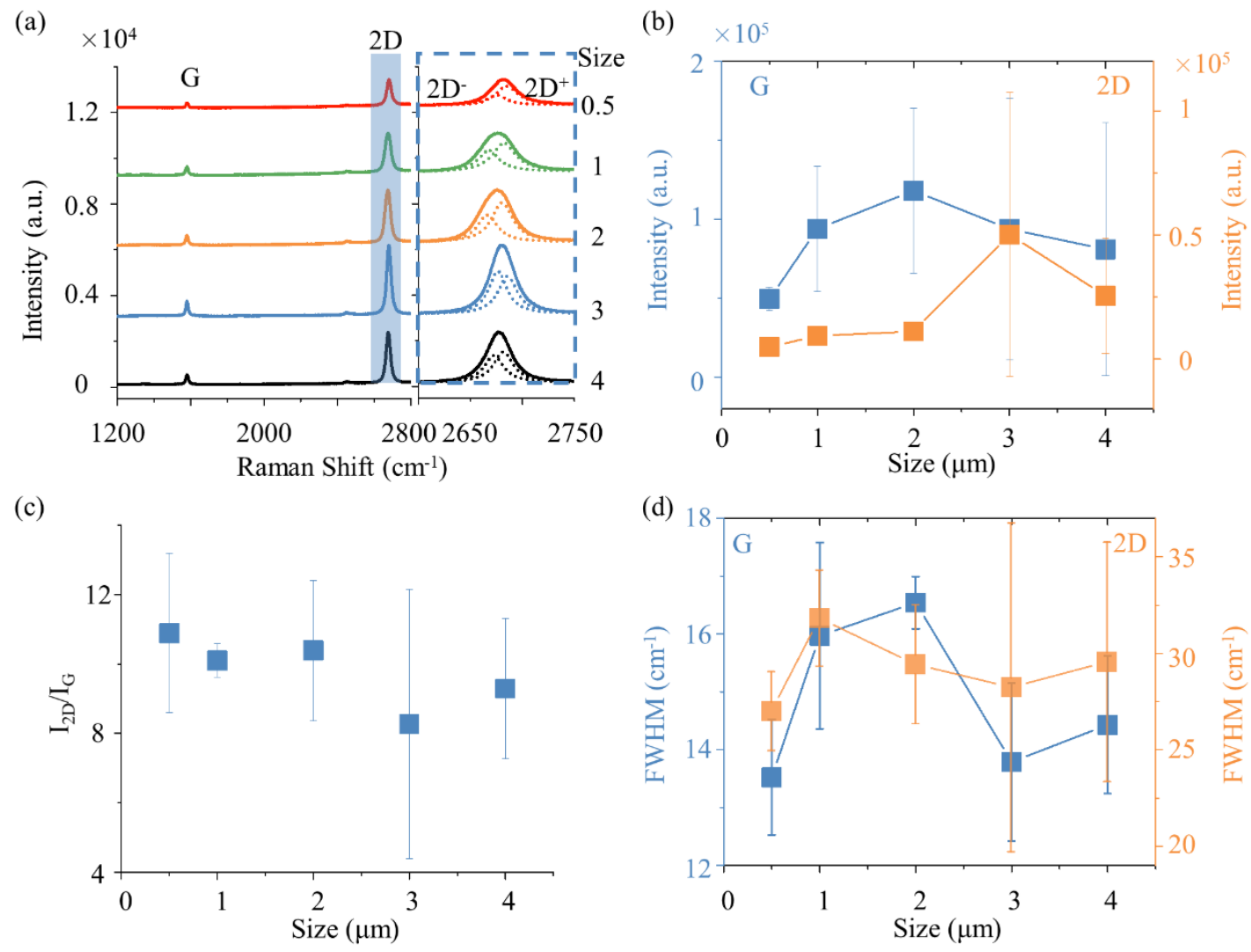

Figure S11. Raman characteristics of suspended graphene on the $\mathrm{Si}_{3} \mathrm{~N}_{4} / \mathrm{SiO}_{2}$ substrate with different size triangular hole structures before helium ions implantation. (a) The 
average spectra and fitted results at 2D peak of suspended graphene. (b) Raman intensities at D and 2D peaks of suspended graphene. (c) Relative Raman intensities between 2D and G peaks of suspended graphene. (d) FWHMs at G and 2D peaks of suspended graphene membrane. Error bars represent s. e. m. $(n=3)$. 